\title{
Comment
}

Neuroepidemiology 2016;46:271-272

DOI: $10.1159 / 000445389$

\section{How Credible Are Meta-Analyses of Risk Factors Based on Observational Studies for Amyotrophic Lateral Sclerosis? A New Insight from an Umbrella Review}

\author{
Ming-Dong Wang ${ }^{a, b}$ Julian Little \\ aSchool of Life Science, Changchun Normal University,

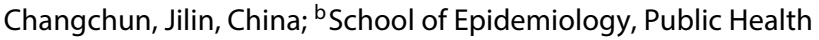 \\ and Preventive Medicine, Faculty of Medicine, University of \\ Ottawa, Ottawa, Ont., Canada
}

Amyotrophic lateral sclerosis (ALS) is a rare multifactorial neurodegenerative disease primarily affecting motor neurons. Based on heritability estimates, it is found that genetic and environmental risk factors play about equally important roles in its etiology $[1,2]$. Polygenic variation is thought to be important in the onset of ALS, whereas monogenic mutations account for a small proportion of cases. No specific environmental risk factors have been firmly associated with ALS so far. Because ALS is rare, it is likely that risk factors identified in single observational studies reflect the play of chance. Synthesizing data from multiple observational studies investigating the same putative risk factors using systematic reviews (SRs) and meta-analyses (MAs) therefore seems logical [3]. Nevertheless, there are at least 3 points worth noting. First, there has been considerable debate about the value of inferences from evidence accumulated in observational studies. Although observational studies are more vulnerable to confounding and bias, they can address questions (i.e. rare events, effects of potentially harmful exposures) that cannot be addressed by randomized controlled trials. They have been considered to provide 'real world' results and thus be more generalizable. Second, there may be issues with the rigor of the review process. These have in part been mitigated by the development of tools to enhance transparency of reporting of both primary studies (STROBE) and SR/ MA (PRISMA) [4], and assessment tools [5]. Third, conclusions may change as evidence accumulates. This has been noted especially for genetic studies, but also for studies of lifestyle and environmental exposures. The concern is being addressed by approaches to update SRs, taking advantage of increased statistical power, likely reductions of the risk of bias in observational studies over time, or both.

\section{KARGER}

() 2016 S. Karger AG, Base

0251-5350/16/0464-0271\$39.50/0

E-Mail karger@karger.com

www.karger.com/ned
Field synopses of genetic association studies were developed as a systematic approach to integrate evidence on gene-disease associations on a scale of more than one or a few genes at a time, grading the evidence based on the criteria of quantity, extent of replication, and protection from bias [6]. Umbrella reviews are emerging as a parallel approach for other types of association studies, with examples relating to risk factors for cancers, diabetes and chronic neurological conditions. They have also been used for evaluating risk factors for neurological conditions - multiple sclerosis [7], Parkinson's disease [8], and now ALS [9]. In the ALS umbrella review, 5 statistics were extracted or calculated for each MA, including total ALS cases in all included studies, exclusion of null value in predictive interval, heterogeneity among included studies, small study effects on the estimate, and excessive number of articles with significance among included studies. Thus, compared to existing quality assessment tools for SR/MA, this umbrella review approach requires the conduct of additional calculations. In addition, the concept of credibility of association between risk factors and ALS was introduced.

In the umbrella review of ALS, 16 unique MAs of risk factors for the disease, investigated in 138 primary observational studies, were included. Only for one of the 16 risk factors, chronic occupational exposure to lead was the evidence of association deemed to be 'credible' [9]. This is an unexpected conclusion, given the fact that almost all these MAs were conducted using similar methods and reported according to the PRISMA guidelines.

Separate SRs/MAs of risk factors for specific diseases may derive from overlapping sets of primary studies. In this umbrella review, this seems to have been the case for some biomarkers, dietary factors, farming, pesticides and perhaps rural living, so that similarity in direction and magnitude of association may reflect the operation of similar biases. These putative risk factors did not satisfy all the criteria for 'credibility' specified. For example, for farming and pesticides, while there were large volumes of evidence in aggregate showing positive significant associations, the MAs included small studies with a higher risk of bias, leading to the reduction of overall credibility. This may be a strength of the umbrella review approach, in that anomalies in patterns of association can become apparent that would not be evident in SR/MA of one risk factor at a time.

Two of the MAs with the greatest volume of evidence, smoking and exposure to extremely low-frequency electromagnetic fields, without an excess number of studies detecting a statistically sig-

Dr. Ming-Dong Wang

School of Life Science

Changchun Normal University

Changchun, Jilin 130032 (China)

E-Mail mdwangforever@yahoo.ca or myang015@uottawa.ca

Dr. Julian Little

School of Epidemiology, Public Health and Preventive Medicine

Faculty of Medicine, University of Ottawa

600 Peter Morand Crescent, Room 101F, Ottawa, ON K1G $5 Z 3$ (Canada)

E-Mail jlittle@uottawa.ca 
nificant association, still showed large $\left(\mathrm{I}^{2}>50 \%\right)$ heterogeneity, underscoring the challenge in the conduct of MA with observational studies.

The approach of Belbasis et al. [9] emphasizes the credibility of the association derived from MA, whereas previously proposed assessment tools for SR/MA have focused on assessment of the risk of bias. However, these methods cannot provide direct evidence of causality, and do not include substantial consideration of biological mechanism, a key component in establishing a causal relationship.

The question arises as to when an SR/MA of observational studies should be conducted? First, what is to be considered is whether the data from included observational studies enable subgroup sensitivity analyses, or if a few included observational studies have conducted and reported analyses that address the effects of potential confounding factors. Second, the assessment of searched studies for conducting SR/MA based on preset inclusion and exclusion criteria is a critical step for investigating potential explanations for heterogeneity of the risk estimate through sensitivity analyses. These sensitivity analyses may be informed by methodological features of the primary observational studies assessed using tools such as that proposed by Downs and Black [10]. Alternatively, an evidence-grading system, mainly based on study design for evidence-based review of risk factors for ALS [11], also could be applied. Third, diverse lines of evidence might be suggestive of a causal relationship, although we recognize that there is debate about the value of the concept of 'biological plausibility' in causal inference.

There appear to be unrealistic expectations that MAs can establish or disprove a causal relationship between risk factors and diseases. Instead, the primary objective of carrying out an SR/MA of observational studies should be to document the volume of evidence, risk of bias and heterogeneity, assess consistency of association, and investigate possible explanations for this.

With the rapid increase of the number of MAs of observational studies, we are facing a real challenge about how to evaluate and use these risk estimates for promoting public health. The expanded evaluation with the umbrella review approach of Belbasis et al. [9] is a welcome contribution.

\section{References}

1 Al-Chalabi A, Fang F, Hanby MF, Leigh PN, Shaw CE, Ye W, Rijsdijk F: An estimate of amyotrophic lateral sclerosis heritability using twin data. J Neurol Neurosurg Psychiatry 2010;81:1324-1326.

2 Wingo TS, Cutler DJ, Yarab N, Kelly CM, Glass JD: The heritability of amyotrophic lateral sclerosis in a clinically ascertained United States research registry. PLoS One 2011;6:e27985.

3 Stroup DF, Berlin JA, Morton SC, Olkin I, Williamson GD, Rennie D, Moher D, Becker BJ, Sipe TA, Thacker SB: Meta-analysis of observational studies in epidemiology: a proposal for reporting. Meta-analysis of observational studies in epidemiology (MOOSE) group. JAMA 2000; 283:2008-2012.

4 Liberati A, Altman DG, Tetzlaff J, Mulrow C, Gotzsche PC, Ioannidis JP, Clarke M, Devereaux PJ, Kleijnen J, Moher D: The PRISMA statement for reporting systematic reviews and meta-analyses of studies that evaluate health care interventions: explanation and elaboration. J Clin Epidemiol 2009;62:e1-e34.

5 Shea BJ, Bouter LM, Peterson J, Boers M, Andersson N, Ortiz Z, Ramsay T, Bai A, Shukla VK, Grimshaw JM: External validation of a measurement tool to assess systematic reviews (AMSTAR). PLoS One 2007;2:e1350.

6 Khoury MJ, Bertram L, Boffetta P, Butterworth AS, Chanock SJ, Dolan SM, Fortier I, Garcia-Closas M, Gwinn M, Higgins JP, Janssens AC, Ostell J, Owen RP, Pagon RA, Rebbeck TR, Rothman N, Bernstein JL, Burton PR, Campbell H, Chockalingam A, Furberg H, Little J, O’Brien TR, Seminara D, Vineis P, Winn DM, Yu W, Ioannidis JP: Genome-wide association studies, field synopses, and the development of the knowledge base on genetic variation and human diseases. Am J Epidemiol 2009;170:269-279.

7 Belbasis L, Bellou V, Evangelou E, Ioannidis JP, Tzoulaki I: Environmental risk factors and multiple sclerosis: an umbrella review of systematic reviews and meta-analyses. Lancet Neurol 2015;14:263-273.

8 Bellou V, Belbasis L, Tzoulaki I, Evangelou E, Ioannidis JP: Environmental risk factors and Parkinson's disease: an umbrella review of meta-analyses. Parkinsonism Relat Disord 2016;23:1-9.

9 Belbasis L, Bellou V, Evangelou E: Environmental risk factors and amyotrophic lateral sclerosis: an umbrella review and critical assessment of current evidence from systematic reviews and meta-analyses of observational studies. Neuroepidemiology 2016;46:96-105.

10 Downs SH, Black N: The feasibility of creating a checklist for the assessment of the methodological quality both of randomised and non-randomised studies of health care interventions. J Epidemiol Community Health 1998;52:377-384.

11 Armon C: An evidence-based medicine approach to the evaluation of the role of exogenous risk factors in sporadic amyotrophic lateral sclerosis. Neuroepidemiology 2003;22:217-228. 